\title{
Forgiveness education in fibromyalgia: A qualitative inquiry
}

\author{
Loren L. Toussaint ${ }^{1}$, Ann Vincent ${ }^{2^{*}}$, Mary O. Whipple ${ }^{2}$, Samantha J. McAllister ${ }^{2}$, \\ Dawn M. Finnie ${ }^{3}$, Julie C. Hathaway ${ }^{4}$, Terry H. $\mathrm{Oh}^{2}$, Kevin C. Fleming ${ }^{2}$, \\ Kristin S. Vickers Douglas ${ }^{3,5}$ \\ ${ }^{1}$ Department of Psychology, Luther College, Decorah, USA \\ ${ }^{2}$ Fibromyalgia and Chronic Fatigue Clinic, Mayo Clinic, Rochester, USA; *Corresponding Author: vincent.ann@mayo.edu \\ ${ }^{3}$ Division of Health Care Policy and Research, Mayo Clinic, Rochester, USA \\ ${ }^{4}$ Patient Education Research, Mayo Clinic, Rochester, USA \\ ${ }^{5}$ Department of Psychiatry and Psychology, Mayo Clinic, Rochester, USA
}

Received 13 November 2013; revised 20 December 2013; accepted 13 January 2014

Copyright (c) 2014 Loren L. Toussaint et al. This is an open access article distributed under the Creative Commons Attribution License, which permits unrestricted use, distribution, and reproduction in any medium, provided the original work is properly cited. In accordance of the Creative Commons Attribution License all Copyrights @ 2014 are reserved for SCIRP and the owner of the intellectual property Loren L. Toussaint et al. All Copyright (c) 2014 are guarded by law and by SCIRP as a guardian.

\section{ABSTRACT}

The purpose of this qualitative study was to assess perspectives on the acceptability and the potential applicability of a forgiveness education in patients with fibromyalgia. The concept and tools of forgiveness were presented to thirteen women (age: 40 - 54 years) with a previous diagnosis of fibromyalgia. Subjects participated in 1 of 2 focus groups following a 90-minute education session in which forgiveness was presented as an emotion-focused coping strategy to deal with interpersonal stressors. Qualitative assessment of focus group discussions reveals 3 themes: 1) forgiveness is healthy and reduces pain, 2) forgiveness is within a patient's personal control, and 3) forgiveness education is similar to other types of patient education and is well received. Our results suggest that forgiveness education is acceptable and feasible in patients with fibromyalgia. This justifies further exploration of forgiveness as an emotion-focused selfmanagement strategy to decrease psychological distress.

\section{KEYWORDS}

Fibromyalgia; Forgiveness Intervention; Pain; Patient Education

\section{INTRODUCTION}

Fibromyalgia is a challenging diagnosis for affected patients, partly because of the elusive nature of an illness with no known cause or cure. The ambiguity related to the diagnosis of fibromyalgia may cause feelings of embarrassment, frustration, guilt, isolation, and shame [1,2]. Furthermore, the constant presence of pain and augmented sensory sensitivity (part of the central sensitization spectrum of symptoms) keeps patients from engaging in life activities (e.g., attending places where they may encounter crowds), and this causes feelings of loss and social isolation [3]. Patients with fibromyalgia attempt to compensate and "fit in" by making extra efforts to demonstrate that their lives are normal, and this extra effort requires great physical and emotional energy expenditure [3]. Physicians who treat patients with fibromyalgia also encounter challenges. Because patients with a diagnosis of fibromyalgia often do not look sick, this can cause doubt in the clinical encounter and leave patients feeling that they are mistrusted and stigmatized $[1,2,4]$. Additionally, patients may have difficulty communicating their symptoms. Together, these factors result in considerable psychological distress, which only exacerbates the burden of illness $[5,6]$. Therefore, an important clinical consideration may be to enhance emotion-based coping by decreasing the psychological burden imposed by interpersonal stressors.

Forgiveness has been conceptualized by Worthington and Scherer [7] as an emotion-focused coping strategy that may promote health; it broadly involves contrasting positive emotions against negative emotions or feelings. Forgiveness-based interventions have demonstrated efficacy in reducing perceived stress, trait anger, and state anger in persons experiencing unforgiveness related to 
interpersonal stress and chronic disease [8,9]. Three of the most common models of forgiveness-based interventions were those developed by Enright, by McCullough and Worthington, and by Luskin [10]. The Enright and Fitzgibbons model [11] uses a 4-stage forgiveness-based intervention: uncovering, deciding, working, and deepening. The model described by McCullough and Worthington [12] is a 5-step process: recalling the injustice, empathizing with one's offender, giving an altruistic gift of forgiveness, committing to forgiveness, and holding on to forgiveness granted to one's offender (REACH). Luskin's model [13] is based on his Forgive for Good method, which focuses on understanding forgiveness, gratefully reframing one's experience of an offense, and developing relaxation methods that facilitate the embodiment of forgiveness through imagery, breathing techniques, and meditation.

We hypothesized that forgiveness could potentially be a useful coping strategy in patients with fibromyalgia [14]. Our rationale was that learning to become more forgiving would enhance a patient's ability to cope with numerous negative emotions (e.g., anger, fear, and depression) that were a consequence of the difficult diagnosis, the challenges of illness, and the stress from navigating the health care system. To test our hypothesis, we designed a brief forgiveness education that incorporated techniques from Luskin [13] and Worthington [15]. In order to obtain patients' perspective regarding the acceptability and potential utility of this education, we conducted a qualitative study in a workshop setting and presented forgiveness as an emotion-focused coping strategy to patients with fibromyalgia.

\section{METHODS}

The study was approved by the Mayo Clinic Institutional Review Board. All study participants provided written, informed consent. All study procedures were conducted from June through October 2011.

\subsection{Participants}

We identified a random sample of patients with fibromyalgia who completed the Fibromyalgia and Chronic Fatigue Clinic Treatment Program in 2010. Participants had to be 30 to 55 years old and live within 100 miles of Rochester, Minnesota. Potential participants were mailed a letter of invitation and instructions to return the letter with their contact information and preference to attend 1 of 2 forgiveness education sessions.

\subsection{Forgiveness Education}

Forgiveness was presented to participants using a video and workbook in a workshop setting. The education started with a definition and discussion of forgiveness. The health benefits of forgiveness, as studied in other chronic disorders (e.g., heart disease, cancer), were described. The facilitator (A.V.) then presented methodology for the practice of forgiveness as an emotion-focused coping strategy. The methodology included recalling and reframing the offense, replacing negative emotions as they arose with the practice of letting go, and simultaneously engaging in relaxation techniques (e.g., deep breathing, purposefully becoming aware of the beauty of nature or the image of someone they loved, or purposefully engaging in simple gratitude exercises). The facilitator then engaged the group in brief exercises of letting go, deep breathing, and feeling gratitude. The workshop concluded with a question-and-answer session.

\subsection{Focus Groups and Qualitative Assessments}

Qualitative assessments included observation of participant engagement during the forgiveness education and analysis of themes that arose as a result of the focus groups.

Observation of Participant Engagement. A member of the research team (D.M.F.) observed the participants during the education as they viewed the presentation, participated in the brief workbook exercises, and engaged in group discussions. A priori, this research member was asked to record observational data to indicate level of participant engagement (e.g., body orientation toward the video, not sleeping, not looking at mobile phone), level of observed emotion (e.g., tearfulness, use of facial tissues), and overall energy in the room (e.g., sense that people were or were not passively participating, tired, or bored).

Focus Groups. Focus groups were conducted by members of the research team who were not part of the education session (K.S.V.D., D.M.F., J.C.H.). For the focus groups, participants were seated in a circle and given general instructions about participation (e.g., actively participate in the discussion, try to respond one at a time, and give examples when possible). The facilitator followed an interview guide (Table 1). Discussions were recorded (audio and video) and transcribed verbatim.

\subsection{Data Analysis}

Focus group data were collected and analyzed by the study investigators with expertise in qualitative research methods; none were involved in the development or delivery of the forgiveness education. Immediately after each focus group, field notes were compared and hypotheses were generated for coding the focus group transcripts. The analytic approach used was similar to that described by Krueger and Casey [16]. Thematic and 
Table 1. Focus group interview guide.

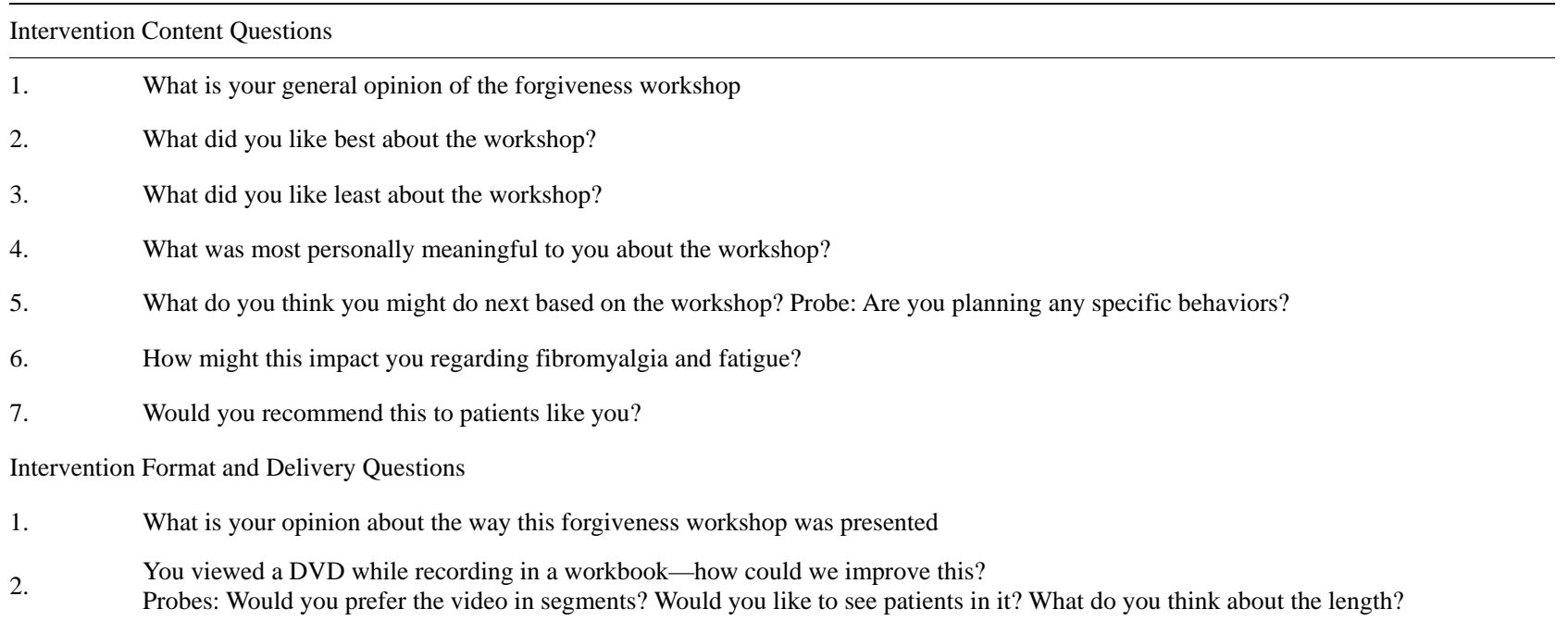

content analysis strategies were used to identify core concepts and to develop categories for coding the data. Predominant themes were identified using methods of content analysis (i.e., using a systematic process of sorting and coding information based on themes) [17]. Investigators independently coded the qualitative data and together identified predominant themes and selected representative quotes for each theme. When discrepancies in coding occurred, the analysts reviewed the qualitative data within the video recording.

\section{RESULTS}

We mailed 184 invitations to participate; of these, 65 responses were returned, with 21 indicating that they would like to participate. Fifteen participants (the first to confirm their appointments) were scheduled for the forgiveness education. Two participants did not attend their scheduled session. Thus, a total of 13 patients completed the study: 5 in the first group and 8 in the second group.

Participants were all women (mean [SD] age, 48 [4.4] years; median [range], 47 [40-54] years). Eleven participants (85\%) were non-Hispanic whites (85\%), 1 (7.7\%) was white (ethnicity unknown), and 1 was non-Hispanic black (7.7\%).

Each education session was 90 minutes long. After the education, participants took a 30-minute break and then reconvened for the focus group. Each focus group lasted approximately 60 minutes (group 1, 49 minutes; group 2, 59 minutes).

\subsection{Observation of Participant Engagement}

Participants were actively engaged during the education. Specifically, all participants were oriented toward the screen during the presentation and toward the workbook and writing when they were asked to do so. There were no instances of participants leaving the room, looking at mobile phones, talking with one another, or directing attention for any duration of time away from intervention materials. From the facial expressions, participants appeared to be fairly intensely concentrating on the material presented. Emotional status of both groups was considered neutral (i.e., no participants had tears in their eyes or used the available facial tissues). Participants were perceived to be sitting calmly and comfortably in their chairs and not demonstrating agitation or restlessness. We did not observe obvious signs of boredom (e.g., yawning, flipping through the workbook, or looking around the room).

\subsection{Themes Related to Educational Content}

In general, the predominant themes were similar across the 2 focus groups. The first group discussed the concept of forgiveness and other aspects of the education content in more depth, whereas the second group emphasized the format and delivery of the education. The combined data from both groups are presented. Three predominant themes emerged from the data.

Theme 1: By Internalizing Stress and Resentment, Physical and Emotional Pain Increases; Forgiveness Helps. Participants understood that holding negative thoughts and feelings weighed them down and that forgiveness may help the process of letting go of negative emotions. They expressed that the forgiveness education made them aware that resentment may be hurting them (instead of the transgressor). They also noted that focusing on more positive thoughts (instead of stressful ones) helped them feel better because it activated coping mechanisms in the body. One participant stated, "I think too, in this day and age, with everyone just popping pills for pain, and not getting to the real reasons... Instead of 
masking with all these drugs, get to the root. I think this helps people identify that pain is more deeply rooted in your emotions and your self-being. You have to fix that."

Theme 2: The Process of Forgiving Helps Maintain Focus on What Can Be Controlled, and Control Is Important in Patients With Chronic Pain. Participants indicated that forgiveness helped them realize that they should not dwell on things that were out of their control; rather, they should focus on what they could control (i.e., themselves). The way that individuals held on to emotions and resentments was viewed as controllable and changeable.

Several participants emphasized that renewing focus on what they could control was particularly important in the context of chronic pain. One participant said, "I need to focus on me and helping me out. Why worry about things that you can't change? So, I can change the way I react to what happened but I can't change what happened." Another stated, "We can't control some of the triggers that might cause us to not feel good... but we can control, like you said, what we do emotionally for ourselves."

Theme 3: The Education Included Familiar Concepts That Were Worthy of Review and Rehearsal. In the focus groups, participants indicated that many concepts were familiar, but they may not have applied them to their illness. Although the content was not novel for the participants, all indicated that the education was still useful because it reminded them to work on this area. Some explained that it was easy to forget about or be distracted from this emotional work and suggested that it must be repeated over time. One person noted that reviewing familiar concepts is often important and likened it to an exercise program, saying "You have good intentions and get started, but then if a bump in the road comes up, it might distract you or you might not do as good job, so you need that reinvestment in it occasionally.”

\subsection{Feedlback about the Education Format and Delivery of Information}

Participants expressed comfort in completing the education in a group setting. Both focus groups agreed that this was preferred over an individual setting, but they thought that more time for group discussion would be beneficial. They did not believe that the content needed to be changed to specifically address fibromyalgia because the concepts were well received and seemed relevant in the context of pain. Some participants described feeling rushed during the workshop and suggested allowing more time for individual workbook exercises to really understand how the general concepts applied to them personally. Some suggested including time for discussion after the education to brainstorm and identify specific actions for individuals in the group. Some sug- gested that having a support person attend the education would be beneficial, whereas others strongly believed that support people should not be included in a patient group, although the content of the education could be shared. Other suggestions pertained to organization of the material for later review. Specifically, some wanted to have more of the video transcribed in the workbook because they were unlikely to watch the video again. One woman stated, "It's nice to have a variety of mediums because people learn differently, and people look for reinforcement material in different ways... It would be nice... to [have the workbook] be more of a mirror, a little bit more of what was in the video in the written.”

The interviewer specifically asked about the language used in the education and about any concerns regarding the concept of forgiveness from a religious or spiritual perspective. (These issues were not covered spontaneously from the group.) Two investigators (K.S.V.D. and J.C.H.) with experience in patient education and plain language noted that some of the language included in the video could be considered psychological jargon. However, focus group participants denied any problems with comprehension and did not recall hearing the words that were used as examples of potential jargon. They emphasized that the overall messages were clear, familiar to them, and congruent with what they knew or believed. Participants denied any concerns about the concept of forgiveness as posing a challenge regarding variability in spiritual and religious beliefs and practices. One participant who stated that she was not a religious person was not offended by the content and did not have concerns about it from this perspective.

\subsection{Applying Forgiveness to Daily Life}

No participant spontaneously described specific actions (e.g., a particular forgiveness strategy) that they planned to take after the education. When asked about their plans, some expressed that they would share the information with a loved one, and others intended to read more from the workbook. Individuals from both groups thought it would be helpful to have this question presented to the group after the education. Participants liked the idea of describing specific steps that could be taken immediately to implement intervention strategies and thought it would be helpful to hear others' plans (i.e., that it would motivate them and provide ideas about applying forgiveness strategies).

\section{DISCUSSION}

Our qualitative study provided a rich description of the participants' perspectives of a brief workshop in which forgiveness was presented as an emotion-focused coping strategy. Analysis of qualitative data revealed 3 important 
themes. First, participants readily accepted forgiveness as an emotion-focused coping strategy and understood the associations between harboring resentment, internalizing stress, and experiencing symptoms. Second, participants welcomed the notion that forgiveness was about controlling personal responses to adversity and injustice and understood that this control was especially relevant in the context of chronic pain. Third, participants found that the forgiveness education reinforced concepts that they had learned in other patient education sessions. Behavioral observations indicated that participants were calm and comfortable, not emotionally aroused, and were actively engaged and attentive. Regarding the delivery method of the education, participants were pleased with the group format and thought the experience was positive overall.

Our findings suggest that forgiveness education warrants further study as an emotion-focused coping modality. Our study has 3 key limitations. First, the sample consisted entirely of women. Future research should evaluate its applicability in groups that include men and assess for gender-based differences in the perception of such interventions. Second, the findings may be influenced by participation bias because these women agreed to attend an extra educational session (beyond the normal routine for patients). We may have inadvertently capitalized on a sample that was unusually eager to learn, and this may not be representative of the general patient population. This could be further studied to assess how a more general population of patients with fibromyalgia would accept the concept of forgiveness. It would be beneficial to evaluate how this educational approach works for patients less accustomed to receiving patient education. Third, our education session was brief, and participants wanted more time for discussion; this will be taken into consideration for future studies.

\section{CONCLUSION}

In summary, our results suggest that forgiveness education is acceptable to patients with fibromyalgia. Our study demonstrates that this education can be easily delivered in a workshop setting and justifies further exploration of forgiveness as an emotion-focused self-management strategy to decrease psychological distress in patients with fibromyalgia.

\section{REFERENCES}

[1] Arnold, L.M., Crofford, L.J., Mease, P.J., Burgess, S.M., Palmer, S.C., Abetz, L. and Martin, S.A. (2008) Patient perspectives on the impact of fibromyalgia. Patient Education and Counseling, 73, 114-120.

http://dx.doi.org/10.1016/j.pec.2008.06.005

[2] Asbring, P. and Narvanen, A.L. (2002) Women's experiences of stigma in relation to chronic fatigue syndrome and fibromyalgia. Qualitative Health Research, 12, 148160.

[3] Sturge-Jacobs, M. (2002) The experience of living with fibromyalgia: Confronting an invisible disability. Research and Theory for Nursing Practice, 16, 19-31. http://dx.doi.org/10.1891/rtnp.16.1.19.52994

[4] Haugli, L., Strand, E. and Finset, A. (2004) How do patients with rheumatic disease experience their relationship with their doctors? A qualitative study of experiences of stress and support in the doctor-patient relationship. $\mathrm{Pa}$ tient Education and Counseling, 52, 169-174. http://dx.doi.org/10.1016/S0738-3991(03)00023-5

[5] Thieme, K., Rose, U., Pinkpank, T., Spies, C., Turk, D.C. and Flor, H. (2006) Psychophysiological responses in patients with fibromyalgia syndrome. Journal of Psychosomatic Research, 61, 671-679. http://dx.doi.org/10.1016/j.jpsychores.2006.07.004

[6] Davis, M.C., Zautra, A.J. and Reich, J.W. (2001) Vulnerability to stress among women in chronic pain from fibromyalgia and osteoarthritis. Annals of Behavioral Medicine, 23, 215-226. http://dx.doi.org/10.1207/S15324796ABM2303_9

[7] Worthington, E.L. and Scherer, M. (2004) Forgiveness is an emotion-focused coping strategy that can reduce health risks and promote health resilience: Theory, review, and hypotheses. Psychology and Health, 19, 385-405. http://dx.doi.org/10.1080/0887044042000196674

[8] Waltman, M.A. (2002) The psychological and physiological effects of forgiveness education in male patients with coronary artery disease. University of Wisconsin, Madison, Waltman.

[9] Harris, A.H., Luskin, F., Norman, S.B., Standard, S., Bruning, J., Evans, S. and Thoresen, C.E. (2006) Effects of a group forgiveness intervention on forgiveness, perceived stress, and trait-anger. Journal of Clinical Psychology, 62, 715-733. http://dx.doi.org/10.1002/jclp.20264

[10] Wade, N.G., Worthington, E.L. and Meyer, J.E. (2005) But do they work? A meta-analysis of group interventions to promote forgiveness. In: Worthington, E.L., Ed., Handbook of Forgiveness (pp. 423-439). Taylor \& Francis.

[11] Enright, R.D. and Fitzgibbons, R.P. (2000) Helping clients forgive: An empirical guide for resolving anger and restoring hope. American Psychological Association.

[12] McCullough, M.E. and Worthington, E.L. (1995) Promoting forgiveness: A comparison of two brief psychoeducational group interventions with a waiting-list control. Counsenling and Values, 40, 55-68. http://dx.doi.org/10.1002/j.2161-007X.1995.tb00387.x

[13] Luskin, F. (2002) Forgive for good: A proven prescription for health and happiness. Harper, San Francisco.

[14] Toussaint, L., Overvold-Ronningen, M., Vincent, A., Luedtke, C., Whipple, M., Schriever, T. and Luskin, F. (2009) Implications of forgiveness enhancement in patients with fibromyalgia and chronic fatigue syndrome. Journal of Health Care Chaplaincy, 16, 123-139. http://dx.doi.org/10.1080/08854726.2010.492713

[15] Worthington, E.L. (2008) Steps to reach forgiveness and 
to reconcile. Pearson Learning Solutions, Boston.

[16] Krueger, R.A. and Casey, M.A. (2009) Focus groups: A practical guide for applied research. 4th ed., Sage Publications, Los Angeles.
[17] Patton, M.Q. (1990) Qualitative evaluation and research methods. 2nd ed., Sage Publications, Newbury Park. 